\title{
Interest consistency can buffer the effect of COVID-19 fear on psychological distress
}

\author{
Masuyama, A. ${ }^{\text {a }}$, Kubo, T. ${ }^{\mathrm{b}}$, Sugawara, D. ${ }^{\mathrm{c}}$, \& Chishima, Y. ${ }^{\mathrm{d}}$
}

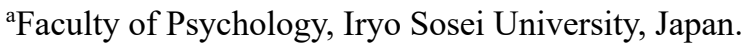

bPsychological Counseling Center, Iryo Sosei University, Japan.

${ }^{c}$ Faculty of Human Sciences, University of Tsukuba, Japan

${ }^{\mathrm{d} D e p a r t m e n t ~ o f ~ P s y c h o l o g y, ~ W i l f r i d ~ L a u r i e r ~ U n i v e r s i t y, ~ C a n a d a ~}$

\section{Corresponding Author}

Akihiro Masuyama, Ph. D.

Affiliation: Faculty of Psychology, Iryo Sosei University, Fukushima, Japan.

Mail Address: ak.masuyama@gmail.com

Address: Chuodai-Iino 5-5-1, Iwaki city, Fukushima, Japan. 970-8551

ORCID ID: https://orcid.org/0000-0001-9014-8129

\section{Co-authors}

Takahiro Kubo, Ph. D.

Affiliation: Psychological Counseling Center, Iryo Sosei University, Fukushima, Japan.

Mail Address: songyou312@gmail.com

Address: Chuodai-Iino 5-5-1, Iwaki city, Fukushima, Japan. 970-8551

ORCID ID: https://orcid.org/0000-0003-0951-1678

Daichi Sugawara, Ph. D.

Affiliation: Faculty of Human Sciences, University of Tsukuba, Japan 
Mail Address: sugawara@human.tsukuba.ac.jp

Address: Tennodai 1-1-1, Tsukuba, Ibaraki, Japan

Chishima Yuta, Ph.D.

Affiliation: Department of Psychology, Wilfrid Laurier University, Warterloo, Ontario, Canada

Mail Address: chishimay@gmail.com

Address: 75 University Ave W, Waterloo, Ontario, Canada

ORCID ID: https://orcid.org/0000-0003-2832-769X

\section{Funding Resource}

This study was partly supported by Research Support Program to Apply the Wisdom of the University to tackle COVID -19 Related Emergency Problems, University of Tsukuba. 


\section{Abstract}

In the context of a recent outbreak of the coronavirus disease (COVID-19), the present study investigated the buffering effect of grit on the relationship between fear of COVID-19 and psychological distress. The data were collected from 224 Japanese participants (98 females; mean age $=46.56, S D=13.41$ ) in July 2020 . The measures used in this study included the Fear of COVID-19 Scale (FCV-19S), Short Grit Scale, and Depression, Anxiety, and Stress Scale 21 (DASS). The results of mediation analyses revealed significant indirect effects of consistency of interest, a major component of grit, on psychological distress; we also found non-significant indirect effects of perseverance of effort, another major component of grit, on psychological distress. These results suggest that consistency of interest buffers the psychological distress induced by fear of COVID-19. Based on these results, it can be concluded that individuals with higher consistency of interest are less likely to experience worsening of their mental health, even if they experience fear of COVID-19 during the pandemic.

Keywords: COVID-19, Grit, Depression, Anxiety, Stress 


\section{Background}

Since March 2019, the outbreak of the coronavirus disease 2019 (COVID-19) has rapidly emerged as a global challenge. According to the World Health Organization (WHO), as of August 4, 2020, the number of confirmed cases of COVID-19 reached 18 million, while the number of death cases exceeded 200,000. To prevent the spread of the virus, governments have implemented various strategies, including promoting remote work, social isolation, and lockdowns. However, the limitations imposed on people's daily lives have led many people to suffer from quarantine-related stress (Mattioli et al., 2020). According to the results reported by Wu et al. (2020), the measures related to COVID-19 pandemic induced physical and mental health-related problems of sleep, worrying, and fear. Likewise, the results of an online survey of a US population revealed that the mean level of depressive symptoms exceeded the clinical cut-off point, suggesting that many people suffer from depression (Fitzpatrick, Harris, \& Drawve, 2020). In this context, it is important to investigate the effects of COVID-19 pandemic on people's psychological aspects and mental health.

One well-known psychological aspect during the current pandemic is the fear of COVID-19. Ever since the development of the Fear of COVID-19 Scale (FCV-19S) by Ahorus et al. (2020), this scale has been translated into many languages, including Arabic (Alyami, etal., 2020), Bangla (Sakib et al., 2020), Hebrew (Bitan et al., 2020), Italian (Soraci et al., 2020), Japanese (Masuyama, Shinkawa, \& Kubo, 2020), Russian (Reznik et al., 2020), Spanish 
(Huarcaya-Victoria, Villarreal-Zegarra, Podestà, \& Luna-Cuadros, 2020), and Turkish (Satici, Gocet-Tekin, Deniz, \& Satici, 2020). Furthermore, many studies have used the FCV-19S to investigate the influence of COVID-19 pandemic on mental health of various populations. Taken together, the results of these studies not only validated the many translated versions of the FCV-19S, but also revealed a strong association between fear of COVID-19 and depression, stress, anxiety, and other variables related to mental health. For instance, Sakib et al. (2020) established that fear of COVID-19 is positively associated with worry about social isolation. Furthermore, Satici et al. (2020) found the indirect mediating effect of psychological distress on the relationship between fear of COVID-19 and life satisfaction. In addition, several previous studies reported the negative effects of fear of COVID-19 on physical and environmental quality of life (e.g., Harper et al., 2020).

However, while this pioneering body of research has broadly investigated the influence of fear of COVID-19 on mental health, less is known about underlying psychological mechanism that explains how the harmful impact of fear of COVID-19 can be eliminated during the pandemic. As many researchers emphasized (Galea, Merchant, \& Lurie, 2020; Torales et al., 2020; Van Bavel et al., 2020), to find the effective ways to improve people's mental health in this unprecedented period, it is necessary to explore the process to ease their psychological distress enhanced by their anxiety of getting the virus.

To explore the mechanism, we focus on a personality trait, especially in the aspect of 
motivation, called "grit". In positive psychology, grit is defined as "perseverance and passion for long-term goals" (Duckworth et al., 2007). In recent years, grit has received much scholarly attention as a psychological trait that can positively affect mental health and social performance (e.g. Hill, Burrow, \& Bronk, 2016; Li et al., 2019; Sharkey et al., 2017). Grit is conceptualized as a psychological personality trait related to motivational perseverance. Structurally, grit is assumed to include the following two major components: (1) consistency of interest in longterm goals and (2) persistence of effort in pursuing those long-term goals (Duckworth \& Quinn, 2009). Previous research has convincingly demonstrated that grid is associated with various aspects of psychological well-being, including life satisfaction (Li et al., 2019), quality of life (Sharkey et al., 2017), and positive emotion (Hill, Burrow, \& Bronk, 2016). Furthermore, Kleiman et al. (2013) found that higher grit predicts the absence of suicidal risk and depressive symptoms. Likewise, Musumari et al. (2018) reported a strong negative association between grit and mental health issues, including depression and anxiety. In the context of these findings, grit can be seen as a protective factor against psychological distress and a predictive factor of psychological wellbeing. Accordingly, grit can be said to be capable of buffering psychological distress. Therefore, in the realities of the current COVID-19 situation, gritty individuals can reasonably be predicted to be better adapted to the pandemic. Yet, to date, none of the previous studies has investigated whether grit would protectively work in the present-day challenging situation. 
Summing up, the previous studies showed that the fear of COVID-19 influenced to psychological distress during current pandemic. Even before the pandemic, grit, involved with perseverance and passion for long-term goal, has been known as a psychological characteristic that stabilizes mental health and can be expected to recover mental health current pandemic situation. However, it is not clear whether grit reduces psychological distress resulting from fear of COVID-19. Thus, the present study aims to explore the buffering effect of grit on the relationships between fear of COVID-19 and psychological distress. Obtaining evidence from this study would contribute to the elaboration of further strategies and policies related to mental health.

\section{Method}

\subsection{Participants and Procedure}

In the present study, we used the dataset from the Primary Survey in Japan (PSJ) conducted within the framework of the Resilience to COVID-19 in Each Region (RE-COVER) project launched in June, 2020. The RE-COVER project aims to reveal recovery factors that can reenergize mental health during the COVID-10 pandemic, with a particular focus on psychological resilience and positive psychological aspects. The RE-COVER project currently targets the following four countries: Japan, the United States, China, and Malaysia (Sugawara et al., 2020; https://osf.io/p56ga/). 
Before proceeding to the broader international arena, the PSJ was initially conducted in Japan. This survey aims to explore the effect of psychological resilience on mental health during the COVID-19 pandemic. The survey was conducted on July 13, 2020, in the form of an online survey hosted by Questant (https://questant.jp). Although an emergency declaration in Japan had been lifted and the strong restriction had been loosened in July, it is assumed that people were still worried about getting infected. The survey was constructed using various psychological measures (e.g., Fear of COVID-19 Scale, the Depression, Anxiety, Stress Scale, Life satisfaction, Sense of Control Scale). The data were collected from a total of 224 participants ( 98 females; mean age $=46.56, S D=13.41$ ). For the present study that aimed to investigate whether grit can buffer the detrimental influence of COVID-related fear on psychological distress, we extracted the participants' responses to the Fear of COVID-19 Scale (FCV-19S), Short Grit Scale, and Depression, Anxiety, and Stress Scale 21 (DASS). The protocol of the PSJ was approved by the author's IRB.

\subsection{Measures}

\section{Fear of COVID-19 Scale}

The Fear of COVID-19 Scale (FCV-19S; Ahorsu et al., 2020) measures the fear towards the COVID-19 infection. The Japanese version of the FCV-19S was previously validated by Masuyama et al. (2020). Although the Japanese version of the FCV-19S was validated in 
adolescents, a subsequent study applied it to the general population and confirmed its validity (Sugawara et al., 2020). The Japanese version of the FCV-19S consists of 7 items to be rated on a 5-point Likert scale. Unlike the one-dimensional construct of the FCV-19S versions in other languages (e.g., Satici et al., 2020; Soraci et al., 2020; Ahorsu et al., 2020), the Japanese version of the FCV-19S is a two-factor construct: emotional response (items 1, 2, 4, 5) and physiological response (items 3, 6, 7). However, because international comparative study (Lin et al., under review) revealed the invariance of one-factor construct of the FCV-19S, we regard the Japanese version FCV-19S as one-dimensional construct. The total score ranges from 7 to 35, with greater scores meaning greater fear of COVID-19. Internal consistency values of FCV19S in the present study was $\alpha=.83$.

\section{Depression, Anxiety, Stress Scale 21}

The Depression, Anxiety, Stress Scale 21 (DASS; Antony, Bieling, Cox, Enns, \& Swinson, 1998) measures participants' psychological distress. The Japanese version of the DASS was previously validated by Adachi and Ueno (2001). The DASS consists of three 7-item subscales: depression, anxiety, and stress. The items are rated on a 4-point Likert scale. The total scores on each subscale range from 1 to 28 , with higher scores indicating more symptoms of psychological distress. Internal consistency values obtained in the present study were as follows: depression, $\alpha=.93$; anxiety, $\alpha=.92$; and stress, $\alpha=.92$.

\section{Short Grit Scale}


The Short Grit Scale measures grit trait (Duckworth \& Quinn, 2009). The Japanese version of the Short Grit Scale was previously validated and confirmed to have excellent reliability and construct validity (Nishikawa, Okugami, \& Amemiya, 2018). The Grit Scale is a two-factor construct that includes perseverance of effort and consistency of interest. Each of the two factors is measured by 4 items to be rated on a 5-point Likert scale. The total scores of each factor range from 4 to 20, with higher score suggesting greater grit trait. Internal consistency values of these two factors in the present study were as follows: perseverance of effort, $\alpha=.85$; consistency of interest, $\alpha=.77$. Although grit is regarded as one component measured by total of these two factors, some researchers have claimed that the two subscales should be separately evaluated (e.g., Credé, Tynan, \& Harms, 2017). Following their suggestion, we decided to use two subscales separately, avoiding summing up.

\subsection{Statistical Analyses}

All variables were computed separately for each subscale or factor. Descriptive statistics and correlation were computed using SPSS, version 26.

To explore whether grit mediates and buffers the relation between fear of COVID-19 and psychological distress (depression, anxiety, and stress), we also conducted mediation analysis. The mediation effect was analyzed by the model 4 setting of the PROCESS macro developed by Hayes (2013). We applied the 95\% bias corrected confidence interval obtained 
from 5,000 bootstrap resampling. As variables in the mediation analyses, we used the total score of FCV-19S, psychological distress score (depression, anxiety, and stress), and traits of grit score (perseverance of effort and consistency of interest). The proposed mediation model was shown in Figure 1.

$<$ Figure 1 $>$

\section{Results}

\subsection{Descriptive Statistics and Correlation Analysis}

Descriptive statistics and the results of correlation analysis are summarized in Table 1 . The results of correlation analysis showed that total score of FCV-19S had significantly positive correlations with psychological distresses, suggesting the strong association with fear of COVID-19 and worsening mental health during the pandemic. For each factor of grit, the results revealed that the perseverance effort negatively correlated with depressive symptoms $(r$ $=-.202, p<.01$ ), while consistency of interest positively correlated with psychological distresses $(r \mathrm{~s}<.215, \mathrm{ps}<.01)$. These results indicate that consistency of interest in grit is strongly associated with fear of COVID-19 and psychological distress, while perseverance effort is related only to depressive symptoms. 
$<$ Table $1>$

\subsection{Mediation Analyses}

Mediation analyses were conducted on the mediated effect of each grit factor (perseverance of effort and consistency of interest) on the relationship between fear of COVID-19 and psychological distress (see Figure1 and Table 2). The results of the analyses on perseverance of effort revealed significant effects of fear of COVID-19 on all types of psychological distress $(\beta \mathrm{s}>.256, p<.05)$ and significant effects of perseverance of effort on depression $(\beta=-.375$, $p<.01,95 \%$ CI: $[-.574,-.176])$. The effect of fear of COVID-19 on perseverance of effort did not reach statistical significance $(\beta=.066,95 \%$ CI: $[-.009, .140])$. Furthermore, the indirect effects of perseverance of effort were not significant on all variables of psychological distress (estimate $=-.02595 \%$ CI: $[-.070, .007]$ for depression, estimate $=-.001[-.017, .008]$ for anxiety, and estimate $=-.001[-.036, .004]$ for distress). Taken together, our results suggest that perseverance of effort does not mediate the relationship between fear of COVID-19 and psychological distress.

Furthermore, the results of mediation analyses on consistency of interest revealed significant effects of fear of COVID-19 on all types of psychological distress $(\beta \mathrm{s}>.195, p<.01)$, as well as significant effects of consistency of interest on psychological distress $(\beta \mathrm{s}<-.200, p$ 
$<.01)$. The latter results suggest that higher consistency of interest is associated with lower psychological distress (including depression, anxiety, and stress). Furthermore, we also observed significant indirect effects of consistency of interest on the relationships between fear of COVID-19 and depression (estimate $=.042 ; 95 \%$ CI: $[.008, .088])$, anxiety (estimate $=.021$; 95\% CI: $[.001, .050])$, and stress (estimate $=.030 ; 95 \%$ CI: $[.004, .066])$. Taken together, these results suggest that the effect of fear of COVID-19 via psychological distress is decreased by consistency of interest.

Overall, our findings on the mediation effects of grit revealed a significant indirect effect of consistency of interest on the relationship between fear of COVID-19 and psychological distresses; however, perseverance of effort was not found to have a significant indirect effect on this relationship. Based on these findings, we can conclude that only one of the two components of grit-namely, consistency of interest - can buffer psychological distress that results from fear of COVID-19.

$<$ Table 2>

\section{Discussion}

The present study aimed to investigate the buffering effect of grit on the relationship 
between fear of COVID-19 and psychological distress during the COVID-19 pandemic. In line with the results of several previous studies (Satici et al., 2020; Voitsidis et al., 2020), we found a significant association between fear of COVID-19 and psychological distress. This finding implies that current pandemic situation, including socio-economic impacts of COVID-19, induces fear of infection and worsens mental health among the general population. Furthermore, our results showed that consistency of interest (one of the two major components of grit) significantly mediates the relation between fear of COVID-19 and psychological distress. This finding suggests that consistency of interest can buffer psychological distress caused by fear of COVID-19. Although we failed to find the mediation effect of perseverance of effort on the relation between fear of COVID-19 and psychological distress, our results provide partial support to the hypothesis about the protective effect of grit during the COVID-19 pandemic.

Although the relationship between grit and mental health has been extensively documented in the literature (Kleiman et al., 2013; Musumari et al., 2018), our results provide novel insights about the relationship between grit and fear of COVID-19. Interestingly, on grit subscale, consistency of interest was significantly correlated with psychological distresses whereas perseverance of effort was not. These results suggest that individuals with high consistency of interest, but not perseverance of effort, experience decreased fear of COVID-19 during the current pandemic situation. In good agreement with our results, Datu, Valdez, and King (2016) reported that consistency of interest—but not perseverance of effort—strongly 
predicted negative-related mental health outcomes. Similarly, von Culin, Tsukayama, and Duckworth (2014) also established a stronger relationship between consistency of interest and pleasure feeling than between perseverance of effort and pleasure feeling. Furthermore, previous studies also found that consistency of interest and perseverance of effort are differentially correlated with mental health (Zhang et al., 2018). Based on these findings, it can be concluded that, compared to perseverance of effort, consistency of interest has a stronger correlation with mental health and other psychological aspects.

We also found that consistency of interest, one of the two major components of grit, has a significant mediation effect on the relationship between fear of COVID-19 and psychological distress. Therefore, consistency of interest can be regarded as a buffer factor against worsening of mental health during the COVID-19 pandemic. Similarly, a previous study found the buffering effect of grit on academic stress during the COVID-19 pandemic (Mosanya, 2020). However, in Mosanya (2020), grit was considered on the whole, i.e. without subdivision into two components. In contrast, in the present study, we focused on two components of grit and found that the individuals with high consistency of interest (but not perseverance of effort) showed decreased psychological distress caused by fear of COVID-19. Taken together, the results of previous studies and our findings highlight the utility of differentiating grit into two components (consistency of interest and perseverance of effort), rather than treating grit as a holistic concept. Our findings about buffering effect of consistency 
of interest imply that people who maintain focus on their projects and pursue their goals, even while facing a worldwide pandemic and its effects to their daily lives, can lessen the harmful effect of fear of COVID-19. It is presumed that consistency of interest can be an important aspect of psychological resilience, and it takes a role of protecting mental health in the pandemic. Individuals with this trait would not be distracted away from their passions because of the COVID-19 pandemic. For instance, a person who continues to play their instruments and pursue their passion for music would be less likely to become depressed from frightening news reports about COVID-19. Future research is needed to further evaluate the way in which consistency of interest lessens distress during a pandemic situation, and perhaps even outside a pandemic.

Furthermore, several previous studies highlighted the important role of positive psychological aspects on mental health and stress induced by the COVID-19 crisis (Barton et al., 2020; Bono, Reil, \& Hescox, 2020). For instance, Y1ldırım and Arslan (2020) found that hope and resilience affect people's subjective wellbeing during the COVID-19 pandemic. Y1ldirım and Arslan (2020) also observed a strong relationship between resilience and preventive behaviors, suggesting that resilience is important for the preservation and protection of mental health during the pandemic. Therefore, positive psychological characteristics are essential for maintaining wellbeing, prevention of mental health issues, and coping with challenges of the current crisis. Viewed from this perspective, our finding that individuals with 
high consistency of interest experience decreased psychological distress make an important contribution to practice. Specifically, based on our results, governments can issue recommendations that would encourage people to engage in consistent attitudes, rather than to explore new opportunities (e.g., starting a new job or hobby). In summary, as already indicated in several previous studies (e.g., Bolier et al., 2013; Sin \& Lyubomirsky, 2009), grit-focused programs should become an integral part of positive psychology interventions to improve mental health during the COVID-19 pandemic.

\section{Limitations}

The present study has several limitations. First, although we theoretically hypothesized the consequences including fear of COVID-19, grit, and psychological distress, the data collection in this study was cross-sectional. Therefore, further longitudinal investigation would be needed to reveal the long-term consequences. Second, since the participants in the present study were recruited from the general population, it remains unclear whether our results can be generalized to specific populations, such as medical workers and patients in need of mental health care. Considering the evidence about the current crisis of mental health among medical workers (Badrfam et al., 2020), as well as COVID-19 survivors (Mazza et al., 2020), further research on the buffering effect of grit in these specific populations is warranted. 


\section{Conclusion}

In the present study, we tested the buffering effect of grit, which was assumed to consist of two major components (consistency of interest and perseverance of effort), on the relationship between fear of COVID-19 and psychological distress. The results of mediation analyses showed that the first of these two components of grit-consistency of interest-has a significant mediation effect on the relation between fear of COVID-19 and psychological distress. These findings suggest that, while fear of COVID-19 can lead to worsening of mental health, this effect can be buffered in individuals with higher consistency of interest.

\section{References}

Adachi K, Ueno T. (2011). The standarization of DASS Japanese version (I). The 24th congress of the Japanese Association of health psychology. Tokyo. pp. 11-12. 
Ahorsu, D. K., Lin, C. Y., Imani, V., Saffari, M., Griffiths, M. D., \& Pakpour, A. H. (2020). The Fear of COVID-19 Scale: Development and Initial Validation. International journal of mental health and addiction, 1-9. Advance online publication. https://doi.org/10.1007/s11469-020-00270-8

Antony, M. M., Bieling, P. J., Cox, B. J., Enns, M. W., \& Swinson, R. P. (1998).

Psychometric properties of the 42-item and 21-item versions of the Depression Anxiety Stress Scales in clinical groups and a community sample. Psychological assessment, 10(2), 176.

Alyami, M., Henning, M., Krägeloh, C. U., \& Alyami, H. (2020). Psychometric evaluation of the Arabic version of the Fear of COVID-19 Scale. International journal of mental health and addiction. https://doi.org/10.1007/s11469-020-00316-x

Barton, M. A., Christianson, M., Myers, C. G., \& Sutcliffe, K. (2020). Resilience in action: Leading for resilience in response to COVID-19. BMJ Leader, 1-3. https://doi.org/10.1136/leader-2020-000260

Badrfam, R., Zandifar, A., \& Arbabi, M. (2020). Mental Health of Medical Workers in COVID-19 Pandemic: Restrictions and Barriers. Journal of Research in Health Sciences, 20(2), e00481. https://doi.org/10.1136/leader-2020-000260

Bitan, D. T., Grossman-Giron, A., Bloch, Y., Mayer, Y., Shiffman, N., \& Mendlovic, S. (2020). Fear of COVID-19 scale: Psychometric characteristics, reliability and 
validity in the Israeli population. Psychiatry Research, 113100.

https://doi.org/10.1016/j.psychres.2020.113100

Bolier, L., Haverman, M., Westerhof, G. J., Riper, H., Smit, F., \& Bohlmeijer, E. (2013).

Positive psychology interventions: a meta-analysis of randomized controlled studies.

BMC public health, 13(1), 119. https://doi.org/10.1186/1471-2458-13-119

Bono, G., Reil, K., \& Hescox, J. (2020). Stress and wellbeing in college students during the COVID-19 pandemic: Can grit and gratitude help? International Journal of Wellbeing, 10(3), 39-57. https://doi.org/10.5502/ijw.v10i3.1331

Credé, M., Tynan, M. C., \& Harms, P. D., 2017. Much ado about grit: A meta-analytic synthesis of the grit literature. J. Pers. Soc. Psychol. 113, 492-511. https://doi.org/10.1037/pspp0000102

Datu, J. A. D., Valdez, J. P. M., \& King, R. B. (2016). Perseverance counts but consistency does not! Validating the short grit scale in a collectivist setting. Current Psychology, 35(1), 121-130. https://doi.org/10.1007/s12144-015-9374-2

Duckworth, A. L., Peterson, C., Matthews, M. D., \& Kelly, D. R. (2007). Grit: Perseverance and Passion for Long-Term Goals. Journal of Personality and Social Psychology, 92(6), 1087-1101. https://doi.org/10.1037/0022-3514.92.6.1087 
Duckworth, A. L., \& Quinn, P. D. (2009). Development and validation of the short Grit Scale (Grit-S). Journal of Personality Assessment, 91(2), 166-174. https://doi.org/10.1080/00223890802634290

Fitzpatrick, K. M., Harris, C., \& Drawve, G. (2020). Fear of COVID-19 and the mental health consequences in America. Psychological Trauma: Theory, Research, Practice, and Policy. http://dx.doi.org/10.1037/tra0000924

Galea, S., Merchant, R. M., \& Lurie, N. (2020). The mental health consequences of COVID19 and physical distancing: The need for prevention and early intervention. JAMA internal medicine, 180(6), 817-818. https://doi.org/10.1001/jamainternmed.2020.1562

Hayes, A. F. (2013). Introduction to mediation, moderation, and conditional process analysis: A regression-based approach. New York: Guilford Press.

Hill, P. L., Burrow, A. L., \& Bronk, K. C. (2016). Persevering with Positivity and Purpose: An Examination of Purpose Commitment and Positive Affect as Predictors of Grit. Journal of Happiness Studies, 17(1), 257-269. https://doi.org/10.1007/s10902-0149593-5

Huarcaya-Victoria, J., Villarreal-Zegarra, D., Podestà, A., \& Luna-Cuadros, M. A. (2020). Psychometric properties of a Spanish version of the Fear of COVID-19 Scale in 
general population of Lima, Peru. International Journal of Mental Health and

Addiction, 1-14. https://doi.org/10.1007/s11469-020-00354-5

Kleiman, E. M., Adams, L. M., Kashdan, T. B., \& Riskind, J. H. (2013). Gratitude and grit indirectly reduce risk of suicidal ideations by enhancing meaning in life: Evidence for a mediated moderation model. Journal of Research in Personality, 47(5), 539-546. https://doi.org/10.1016/j.jrp.2013.04.007

Li, J., Fang, M., Wang, W., Sun, G., \& Cheng, Z. (2018). The influence of grit on life satisfaction: Self-esteem as a mediator. Psychologica Belgica, 58(1), 51-66. https://doi.org/10.5334/pb.400

Lin, C., Hou, W., Mamun, M., Aparecido, S., José, B. Y., ..., Pakpour, A. H., under review. Fear of COVID-19 Scale (FCV-19S) across countries: Measurement invariance issues. Nursing Open.

Mattioli, A. V., Nasi, M., Cocchi, C., \& Farinetti, A. (2020). COVID-19 outbreak: impact of the quarantine-induced stress on cardiovascular disease risk burden. https://doi.org/10.2217/fca-2020-0055.

Masuyama, A., Shinkawa, H. \& Kubo, T. Validation and Psychometric Properties of the Japanese Version of the Fear of COVID-19 Scale Among Adolescents. International Journal of Mental Health and Addiction (2020). https://doi.org/10.1007/s11469-02000368-z 
Mazza, M. G., De Lorenzo, R., Conte, C., Poletti, S., Vai, B., Bollettini, I., ... \& Benedetti, F. (2020). Anxiety and depression in COVID-19 survivors: Role of inflammatory and clinical predictors. Brain, behavior, and immunity, 89, 594-600. https://doi.org/10.1016/j.bbi.2020.07.037

Mosanya, M., 2020. Buffering academic stress during the COVID-19 pandemic related social isolation: Grit and growth mindset as protective factors against the impact of loneliness. International journal of Applied Positive Psycholgy https://doi.org/10.1007/s41042-020-00043-7

Musumari, P. M., Tangmunkongvorakul, A., Srithanaviboonchai, K., Techasrivichien, T., Suguimoto, S. P., Ono-Kihara, M., \& Kihara, M. (2018). Grit is associated with lower level of depression and anxiety among university students in Chiang Mai, Thailand: A cross-sectional study. PLoS ONE, 13(12), 1-16. https://doi.org/10.1371/journal.pone.0209121

Nishikawa,K., Okugami, S., \& Amemiya, T. (2015). Development of the Japanese Short Grit Scale (Grit-S). Japanese Journal of Personality, 24, 167-169.

Reznik, A., Gritsenko, V., Konstantinov, V., Khamenka, N., \& Isralowitz, R. (2020). COVID19 fear in Eastern Europe: Validation of the Fear of COVID-19 Scale. International journal of mental health and addiction, 1. https://doi.org/10.1007/s11469-020-00283- 
Sakib, N., Bhuiyan, A. I., Hossain, S., Al Mamun, F., Hosen, I., Abdullah, A. H., ... \& Sikder, M. T. (2020). Psychometric validation of the Bangla Fear of COVID-19 Scale: confirmatory factor analysis and Rasch analysis. International Journal of Mental Health and Addiction. https://doi.org/10.1007/s11469-020-00289-x

Satici, B., Gocet-Tekin, E., Deniz, M. E., \& Satici, S. A. (2020). Adaptation of the Fear of COVID-19 Scale: its association with psychological distress and life satisfaction in Turkey. International Journal of Mental Health and Addiction. https://doi.org/10.1007/s11469-020-00294-0

Sin, N. L., \& Lyubomirsky, S. (2009). Enhancing well-being and alleviating depressive symptoms with positive psychology interventions: A practice-friendly meta-analysis. Journal of clinical psychology, 65(5), 467-487. https://doi.org/10.1002/jclp.20593

Soraci, P., Ferrari, A., Abbiati, F. A., Del Fante, E., De Pace, R., Urso, A., \& Griffiths, M. D. (2020). Validation and psychometric evaluation of the Italian version of the Fear of COVID-19 Scale. International Journal of Mental Health and Addiction. https://doi.org/10.1007/s11469-020-00277-1

Sharkey, C. M., Bakula, D. M., Gamwell, K. L., Mullins, A. J., Chaney, J. M., \& Mullins, L. L. (2017). The Role of Grit in College Student Health Care Management Skills and HealthRelated Quality of Life. Journal of Pediatric Psychology, 42(9), 952-961. https://doi.org/10.1093/jpepsy/jsx073 
Sugawara, D., Masuyama, A., \& Kubo, T. (2020, August 24). RE-COVER: REsilience for COVid-19 in Each Region. https://doi.org/10.17605/OSF.IO/P56GA

Takebashi, H., Higuchi, O., Ozaki, Y., Watanabe, T., \& Toyosawa, J. (2018). Reliability and validity of the Japanese version of the Grit Scale. Japanese Psychological Research, 89, 580-590.

Torales, J., O’Higgins, M., Castaldelli-Maia, J. M., \& Ventriglio, A. (2020). The outbreak of COVID-19 coronavirus and its impact on global mental health. International Journal of Social Psychiatry, https://doi.org 10.1177/0020764020915212.

Van Bavel, J. J., Baicker, K., Boggio, P. S., Capraro, V., Cichocka, A., Cikara, M., ... \& Drury, J. (2020). Using social and behavioural science to support COVID-19 pandemic response. Nature Human Behaviour, 1-12. https://doi.org/10.1038/s41562-020-0884-z

Voitsidis, P., Nikopoulou, V. A., Holeva, V., Parlapani, E., Sereslis, K., Tsipropoulou, V., ... \& Diakogiannis, I. (2020). The mediating role of fear of COVID-19 in the relationship between intolerance of uncertainty and depression. Psychology and Psychotherapy: Theory, Research and Practice. https://doi.org/10.1111/papt.12315

Von Culin, K. R., Tsukayama, E., \& Duckworth, A. L. (2014). Unpacking grit: Motivational correlates of perseverance and passion for long-term goals. The Journal of Positive Psychology, 9(4), 306-312. https://doi.org/10.1080/17439760.2014.898320

Wu, W., Zhang, Y., Wang, P., Zhang, L., Wang, G., Lei, G., ... \& Huang, F. (2020). 
Psychological stress of medical staffs during outbreak of COVID-19 and adjustment strategy. Journal of Medical Virology. https://doi.org/10.1002/jmv.25914

World Health Organization. (2020, August 4). Coronavirus disease (COVID-2019) situation reports. https://www.who.int/emergencies/diseases/novel-coronavirus2019/situation-reports

Zhang, M. X., Mou, N. L., Tong, K. K., \& Wu, A. (2018). Investigation of the effects of purpose in life, grit, gratitude, and school belonging on mental distress among Chinese emerging adults. International journal of environmental research and public health, 15(10), 2147. https://doi.org/10.3390/ijerph15102147 


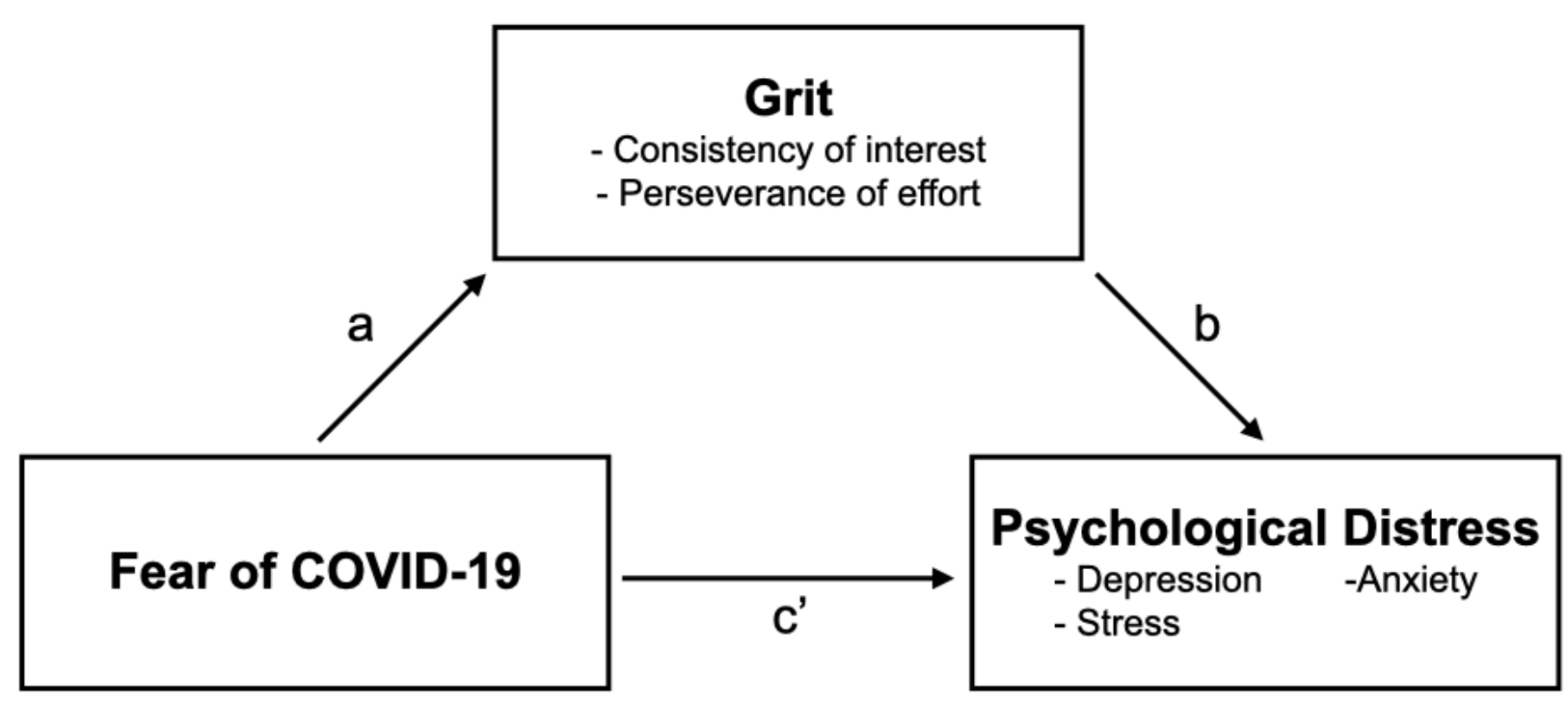

Figure 1. Proposed mediation model of this study. 
Table 1. Descriptive statistics and Correlations of each valuables.

\begin{tabular}{|c|c|c|c|c|c|c|c|c|}
\hline & & $M$ & $S D$ & 1 & 2 & 3 & 4 & 5 \\
\hline 1 & FCV-19S & 20.84 & 5.39 & - & & & & \\
\hline \multicolumn{9}{|c|}{ DASS } \\
\hline 2 & Depression & 11.08 & 4.82 & $.262 *$ & - & & & \\
\hline 3 & Anxiety & 9.20 & 3.71 & $.367 *$ & $.761 *$ & - & & \\
\hline 4 & Stress & 10.78 & 4.52 & $.392 *$ & $.843 *$ & $.838 *$ & - & \\
\hline \multicolumn{9}{|c|}{ Short Grit Scale } \\
\hline 5 & Perseverance of effort & 11.23 & 3.03 & .116 & $-.202 *$ & .026 & -.053 & - \\
\hline 6 & Consistency of interest & 11.51 & 2.70 & $-.205 *$ & $-.270 *$ & $-.215 *$ & $-.243 *$ & .115 \\
\hline
\end{tabular}

Note. FCV-19S: Fear of COVID-19 Scale, DASS: Depression, Anxiety, and Stress Scale.

$* p<.01$ 
Table 2. Results of mediation analyses.

\begin{tabular}{|c|c|c|c|c|c|c|c|c|c|}
\hline & \multicolumn{3}{|c|}{ Depression } & \multicolumn{3}{|c|}{ Anxiety } & \multicolumn{3}{|c|}{ Stress } \\
\hline & $\beta(\mathrm{SE})$ & $t$ & $95 \% \mathrm{CI}$ & $\beta(\mathrm{SE})$ & $t$ & $95 \% \mathrm{CI}$ & $\beta(\mathrm{SE})$ & $t$ & $95 \% \mathrm{CI}$ \\
\hline \multicolumn{10}{|l|}{ Mediator: Perseverance of effort } \\
\hline \multicolumn{10}{|l|}{ Variables } \\
\hline Fear of COVID-19 (c') & $.261(.057)$ & $4.566^{* *}$ & {$[.148, .374]$} & $.256(.044)$ & $5.862 * *$ & {$[.170, .342]$} & $.341(.052)$ & $6.512^{*}$ & {$[.238, .444]$} \\
\hline Perseverance of effort (b) & $-.375(.101)$ & $3.717 * *$ & $\begin{array}{l}{[-.574} \\
-.176]\end{array}$ & $-.020(.077)$ & 0.269 & {$[-.173, .131]$} & $-.148(.092)$ & 1.603 & {$[-.330, .034]$} \\
\hline Indirect effect & $-.025(.020)$ & & {$[-.070, .007]$} & $-.001(.006)$ & & {$[-.017, .008]$} & $-.001(.010)$ & & {$[-.036, .004]$} \\
\hline \multicolumn{10}{|l|}{ Mediator: consistency of interest } \\
\hline \multicolumn{10}{|l|}{ Variables } \\
\hline Fear of COVID-19 (c') & $.195(.058)$ & $3.343 * *$ & {$[.080, .309]$} & $.240(.044)$ & $5.341 * *$ & {$[.148, .320]$} & $.302(.053)$ & $5.741 * *$ & {$[.198, .405]$} \\
\hline Consistency of interest (b) & $-.404(.116)$ & $3.502 * *$ & $\begin{array}{l}{[-.632} \\
-.177]\end{array}$ & $-.200(.087)$ & $2.304^{*}$ & $\begin{array}{l}{[-.371} \\
-.029]\end{array}$ & $-.285(.104)$ & $2.736 * *$ & $\begin{array}{l}{[-.491} \\
-.080]\end{array}$ \\
\hline \multirow[t]{3}{*}{ Indirect effect } & $.042(.021)$ & & {$[.008, .088]$} & $.021(.013)$ & & {$[.001, .050]$} & $.030(.016)$ & & {$[.004, .066]$} \\
\hline & \multicolumn{3}{|c|}{ Perseverance of effort } & \multicolumn{3}{|c|}{ Consistency of interest } & & & \\
\hline & $\beta(\mathrm{SE})$ & $t$ & $95 \% \mathrm{CI}$ & $\beta(\mathrm{SE})$ & $t$ & $95 \% \mathrm{CI}$ & & & \\
\hline Fear of COVID-19 (a) & $.066(.038)$ & 1.739 & {$[-.009, .140]$} & $-.103(0.33)$ & $3.120^{*}$ & {$[-.169,-.038]$} & & & \\
\hline
\end{tabular}

$* p<.05, * * p<.01$ 